EMBRYARIDDLE
Aeronautical University

SCHOLARLY COMMONS

\section{International Journal of Aviation,} Aeronautics, and Aerospace

7-19-2016

\title{
Increasing Collegiate Flight Training Fleet Utilization Through the Use of an Aircraft Assignment Algorithm
}

\author{
John H. Mott \\ Purdue University, jhmott@purdue.edu \\ Daniel Henao \\ Universidad Nacional de Colombia - Sede Medellin, dhenao1991@gmail.com \\ Mitchell S. Hodgen \\ Purdue University, mitchhodgen@gmail.com \\ Darcy M. Bullock \\ Purdue University, darcy@purdue.edu
}

Follow this and additional works at: https://commons.erau.edu/ijaaa

Part of the Management and Operations Commons

\section{Scholarly Commons Citation}

Mott, J. H., Henao, D., Hodgen, M. S., \& Bullock, D. M. (2016). Increasing Collegiate Flight Training Fleet Utilization Through the Use of an Aircraft Assignment Algorithm. International Journal of Aviation, Aeronautics, and Aerospace, 3(3). https://doi.org/10.15394/ijaaa.2016.1131

This Article is brought to you for free and open access by the Journals at Scholarly Commons. It has been accepted for inclusion in International Journal of Aviation, Aeronautics, and Aerospace by an authorized administrator of Scholarly Commons. For more information, please contact commons@erau.edu. 
Among those factors that exert considerable influence on the efficiency with which aircraft within a training fleet are operated, scheduling is instrumental. A particular Midwestern university uses 16 Cirrus SR-20 aircraft in the first two years of the curriculum in its Professional Flight program; these aircraft account for the majority of the total student flight time in that program's primary flight courses. Avery (2014) calculated an average utilization rate for the school's Cirrus fleet of $24 \%$. According to Mott and Bullock (2015), who conducted a more recent examination of utilization data for the Cirrus portion of the school's primary training fleet, the Cirrus aircraft exhibited an average aircraft utilization rate of $26.25 \%$ during normal operating times of the training facility over a period extending back to the beginning of the Fall 2014 semester. An improvement in the efficiency of the scheduling of the Cirrus fleet would allow the resulting excess capacity of schedulable time to be used to support an increase in enrollment in the flight training program. By spreading the fixed costs of the program across a larger number of students, the course fees for the program could be potentially decreased, improving the overall affordability of the program and assisting the University in meeting its president's goal of improving affordability for all students (Daniels, 2014, 2015). The problem under consideration, therefore, is related to the determination of an optimal sequence of flights made by a fleet of general aviation training aircraft such that cumulative turn time (the amount of time that aircraft remain on the ground after a landing and before a subsequent takeoff) of the fleet is minimized. By minimizing the cumulative turn time, the overall fleet utilization rate will, ceteris paribus, increase.

Bazargan (2012) presented an excellent overview of the use of linear programming techniques for a wide range of problems encountered in the airline industry. The basic problem of assigning airline aircraft types to particular routes to achieve minimum cost under an assumption of fixed demand, however, was addressed as early as the 1950s by Ferguson and Dantzig (1954). This work introduced the use of linear programming as a means for solving the fundamental aircraft routing problem. The authors later extended this research to include the case in which demand is uncertain (Ferguson \& Dantzig, 1956). Abara (1989) described the application of these techniques to a practical fleet assignment problem at American Airlines, while another team of researchers did the same at Delta Air Lines (Subramanian, Scheff, Quillinan, Wiper, \& Marsten, 1994). A limitation inherent in all of these procedures, however, is that they assign aircraft types to flights, as opposed to assigning individual aircraft to those flights. Hane et al. (1995) recognized this limitation, and noted that "because the model does not know the previous or next flights to which the aircraft will connect (excepting connections derived from required hookups or islands), many decisions needed to implement a schedule are postponed" (Hane et al., 1995, p. 231). 
A set partitioning approach to the fleet assignment problem (Desaulniers, Desrosiers, Dumas, Solomon, \& Soumis, 1997) addressed the limitation noted by Hane et al., by suggesting that the definition of a flight leg could be extended "to include sequences of consecutive operational flight legs that must be assigned to the same aircraft as required by the airline" (p. 847). This requires the introduction of only one additional covering constraint in the problem formulation. El Moudani and Mora-Camino (2000) developed a solution scheme using a dynamic programming approach to solve the fleet assignment problem, coupled with a heuristic technique to solve the embedded aircraft assignment / maintenance schedule problem. Jarrah, Goodstein and Narasimhan (2000) further suggested that the re-fleeting solution, whereby aircraft-level adjustments are made to address constraints that are not satisfactorily reflected in the initial model, could in fact be effectively integrated in the fleet assignment computation. Grönkvist (2005) developed a hybrid approach to the tail assignment problem, which combines fleet and aircraft-level methodologies into an overall solution applicable at the airline level. Salazar-González (2014) applied a heuristic solution to the overall fleet-aircraft-crew problem to realworld data generated by a European carrier. In addition, Zhu (2006) examined the use of two-stage stochastic mixed integer solutions to fleet problems in an effort to incorporate the use of random variables to improve the existing deterministic solutions.

In an application for individual aircraft in smaller fleets, researchers at the Georgia Institute of Technology utilized a set partitioning model to solve both the aircraft routing problem and the crew assignment problem for an on-demand operation (Yao, Zhao, Ergun, \& Johnson, 2005). Jacobs (2014) demonstrated how a linear optimization approach could be applied to the scheduling of military training flights.

While a substantial amount of research related to the use of linear programming methods in the optimal scheduling of airline aircraft has been conducted, relatively little investigation into a similar application of these tools in large flight training operations has been performed. Bazargan and McGrath (2003) applied a discrete event simulation model to the maintenance scheduling problem in a flight training fleet in an effort to address aircraft availability at the operational level. Their implementation of a new working schedule at Embry-Riddle Aeronautical University resulted in decreased maintenance downtime and improved labor utilization. Their work, however, addressed the supply side of the equation, as opposed to the demand side. Collegiate flight training programs, in particular, tend to have a unique set of optimization constraints that must be considered when performing dispatch scheduling optimization. Among these are the scheduling of flight training times around standard class periods during the day, the greater variability of aircraft availability due to maintenance issues, and the mix of short, local training flights and longer cross-country flights, each of which have substantially different 
mean durations with relatively narrow standard deviations. It is readily apparent, then, that a unique set of optimization constraints applies to the use of linear programming techniques in these specific operations.

The research described herein focused on the application of typical linear programming methods to the solution of the aircraft-level assignment problem with the incorporation of the unique constraints found in a typical collegiate flight training operation. This application will consist of three separate models and algorithms: scheduling, simulation, and flight cancellation.

\section{Method}

While aircraft utilization is dependent upon the exposure basis over which it is calculated, and can be defined in different ways, it is apparent that there is an opportunity to increase the operational efficiency of the training fleet under consideration through improvements in the scheduling process. The exposure basis used for the present calculations covers a period from 7:30 am to 7:30 pm (with an exception described below), Monday through Saturday, during the regular academic semester and excluding academic holidays.

The utilization rate is determined primarily by the scheduling and dispatching of the available training aircraft. Thirteen of the Cirrus aircraft are used for primary training and are considered by the optimization model. There are two categories of flights: local and cross-country. Local flights depart the airport, have a mean duration of 1.27 hours, and return to the airport. The basic purpose of these flights is to train students in the fundamental maneuvers and procedures required to operate aircraft. On the other hand, on a cross-country flight, the student departs the local airport, lands at a different airport at least 50 nautical miles away from the point of departure, departs again, lands and departs from a second airport, and returns to the departure airport. That flight trains a student in the procedures needed when flying from an origin to a destination; i.e., weather checks, weight and balance calculations, route selection, etc. The mean duration of these flights is 2.29 hours, with a greater variance of duration than that of the local flights.

\section{Modeling the Scheduling Process}

The institution currently does not have an explicit scheduling policy that aims to maximize aircraft flight time (and therefore minimize ground time). The underlying assumption in the research presented herein is based on the consideration that if aircraft turn times are decreased by an optimal assignment of scheduled flights to each aircraft, the overall utilization rate will increase. It is important to note that the institutional safety culture is such that minimization of ground times shall not be allowed to compromise operational safety in any manner. 


\section{Assumptions of the Schedule Model}

The following assumptions are incorporated in the linear programming model:

- The number of available aircraft is constant. This means that if an aircraft experiences a mechanical problem rendering it unairworthy during the day, it can be replaced by a "reserved" aircraft that will serve as a substitute for the remainder of the day, or for the time during which the first aircraft is out of service. Initially, the model assumes that there are 16 aircraft; three of these are assumed to be reserved and are unassigned by the model. This does not imply that the reserved aircraft will not fly; it simply means that these aircraft are not scheduled by the model and are available for unscheduled flights.

- There are five available two-hour schedule blocks, beginning at 7:30 am, per aircraft per day when standard time is in effect; that number increases to six during daylight savings time. Local flights are required to depart and arrive within the same two-hour schedule block. Because this two-hour period is impractical for the majority of cross-country flights, such flights must occur within two consecutive two-hour blocks.

- The model will assign 13 flights by default in each two-hour block.

- The departure times of all flights are calculated before the optimization phase. The term departure time is otherwise known as the engine start time, the time when the aircraft engine is started prior to the aircraft leaving the ramp before a flight. Similarly, the arrival time is understood to be the time at which the aircraft engine is shut down after the aircraft returns to the ramp following a flight.

- The turn time between an arriving flight and a departing flight is calculated as the difference between the departure time of the outbound flight and the arrival time of the inbound flight.

- The flight lengths for both local and cross-country flights are considered deterministic, and are represented by mean times of the respective type as calculated from historical data recorded by the Garmin G1000 avionics platforms with which the training aircraft are equipped. For local flights, the mean is 1.27 hours $(N=4,293, \sigma=0.214)$. For crosscountry flights, the mean is 2.29 hours $(N=1,084, \sigma=0.46)$. The flight lengths are measured from engine start to shutdown.

- The first departure of the day is scheduled at 7:45 am and the first departure of each of the subsequent flight blocks is scheduled 10 minutes after the beginning of the block (9:40 am, 11:40 am, etc.). Each subsequent departure in a particular flight block is staggered five minutes from the previous departure.

- A minimum acceptable turn time is required in order to assign an arriving and a departing flight to the same aircraft as a result of required pre- and post-flight checks of the aircraft. The real turn time, then, will 
be greater than that determined by the model because of the difference in the definitions of departure and arrival times. Figure 1 depicts the difference between the real turn times and those in the model formulation.

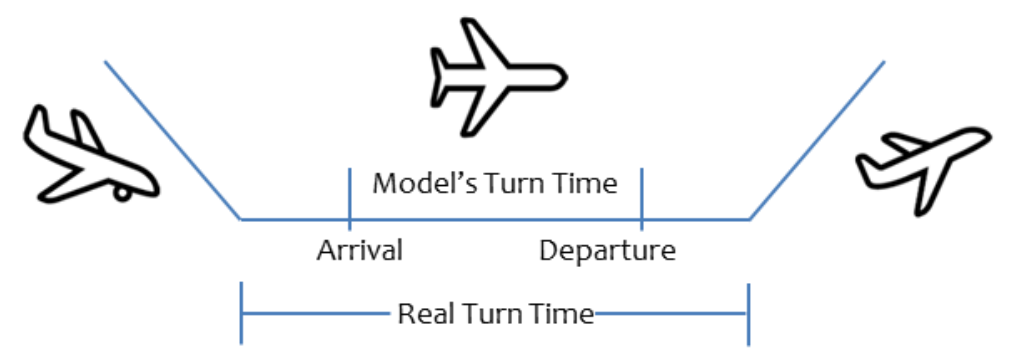

Figure 1. Comparison between the real and the model-based turn times.

\section{Formulating the Model}

The scheduling model presented herein is based on the work of Freling, Pinto Paixao, and Wagelmans (1995). Let $I$ be a set consisting of scheduled flights, where $I=\{1,2,3, \ldots, n\}$, and let $J$ be an alias of $I$. Let $T$ be the set of schedulable time blocks, such that $T=\{1,2,3, \ldots, 6\}$. Let $B_{\mathrm{i}}$ represent the corresponding schedule block for flight $i$. Then

$$
B_{i}=\left\{\begin{array}{l}
1 \text { if flight } i \text { belongs to the 7: } 30 \text { block } \\
2 \text { if flight } i \text { belongs to the 9: } 30 \text { block } \\
3 \text { if flight } i \text { belongs to the 11: } 30 \text { block } \\
4 \text { if flight } i \text { belongs to the 13: } 30 \text { block } \\
5 \text { if flight } i \text { belongs to the 15: } 30 \text { block } \\
6 \text { if flight } i \text { belongs to the 17:30 block }
\end{array} \forall i \in I\right.
$$

Let $V_{i}$ be the expected length of flight $i$, and let $N_{t}$ represent the number of scheduled flights in schedule block $t$. Then

$$
N_{t}=\frac{\sum_{j \mid B_{j}=t} B_{j}}{t} \quad \forall t \in T
$$

Let $t_{s}$ be the minimum staggering time between departures. Then $D_{i}$, the departure time of flight $i$, is given by

$$
D_{i}=\left\{\begin{array}{c}
7.75+t_{s} \times(i-1) \text { if } B_{i}=1 \\
7.67+2\left(B_{i}-1\right)+t_{s}\left(i-\sum_{t=1}^{B_{i}-1} N_{t}-1\right) \text { if } B_{i} \geq 2
\end{array} \quad \forall i \in I\right.
$$

Let $t_{i j}$ be the turn time between flights $i$ and $j$. Then 


$$
t_{i j}=D_{j}-\left(D_{i}+V_{i}\right) \quad \forall i \forall j \in I
$$

Finally, let $t_{\min }$ represent the minimum turn time, and $N$ represent the total number of available aircraft.

As noted previously, the turn time specified in Equation (4) is calculated as the difference between the departure time of the outbound flight and the arrival time of the incoming flight. The arrival time is the quantity $D_{i}+V_{i}$, and is simply the departure time plus the flight length. Note that all times in the program are measured in decimal format.

Now let

$$
\begin{gathered}
F_{j}=\left\{\begin{array}{r}
1 \text { if flight } j \text { is the first flight flown by an aircraft } \\
0 \text { otherwise }
\end{array}\right. \\
X_{i j}=\left\{\begin{array}{r}
1 \text { if flight } j \text { is flown after flight } i \text { in the same aircraft } \\
0 \text { otherwise }
\end{array}\right. \\
L_{j}=\left\{\begin{array}{r}
1 \text { if flight } j \text { is the last flight flown by an aircraft } \\
0 \text { otherwise }
\end{array}\right.
\end{gathered}
$$

Note that these variables are binary. When the solution of the problem has been determined, the values of the decision variables will assist in the construction of the sequences of flights that each aircraft will make. To illustrate, suppose that $F_{2}=X_{23}=L_{3}=1$. The interpretation of those variables is as follows: A specific aircraft has been assigned to start the day with flight number 2. After flying that flight, the aircraft will fly flight number 3, which is the last flight assigned to that aircraft that day.

We wish to minimize the objective function $Z$, which represents the overall turn time. $Z$ can be written as

$$
Z=M \sum_{i} F_{i}+\sum_{i} \sum_{j} t_{i j} X_{i j}
$$

where $M$ is large. Equation (8) shows that the objective function consists of two summations; the first summation penalizes the usage of aircraft (as an algebraic strategy that aims to accommodate all the flights with the least possible number of aircraft), while the second aggregates the turn times of all the flights (the $t_{i j}$ ) that are connected in the same aircraft, which is represented by a value of 1 of the $X_{i j}$ variable. Notice that if $X_{i j}=0$ then the corresponding turn time is not considered in the summation.

At this point, it is also important to realize that the assignment of flights to aircraft depends on the turn times of those aircraft, which in turn depends on the departure times; therefore, the departure times are an important parameter. The manner in which the departure times are assigned is thus crucial to the problem formulation. The optimization constraints are specified as follows:

$$
F_{j}+\sum_{i} X_{i j}=1 \forall j \in I
$$




$$
\begin{gathered}
\sum_{j} X_{i j}+L_{i}=1 \forall i \in I \\
\sum_{i} F_{i} \leq N \\
X_{i j}=0 \text { if } t_{i j}<t_{\text {min }} \text { or } \sum_{i} \sum_{j} X_{i j} \mid t_{i j}<0=0 \\
X_{i j}, F_{j}, L_{j} \in\{0,1\} \forall i \forall j \in I
\end{gathered}
$$

Equation (9) ensures that each flight is either the first one flown by an aircraft or a subsequent flight after any possible preceding flight. Equation (10) ensures that each flight is either followed by a subsequent flight or is the last flight flown by an aircraft. Equation (11) is written in order to use fewer than the number (or the exact number) of available aircraft. Equation (12) guarantees that only feasible connections between flights are considered when connecting flights. Finally, Equation (13) classifies the decision variables as binary variables.

Note that the actual number of aircraft used is calculated indirectly as the sum of the $F$ variables that are equal to 1 . Note also that the assignment of flights to aircraft with specific tail numbers is made by the user when interpreting the values of the decision variables and is based on the maintenance prioritization, as explained in a subsequent section.

It is readily apparent that the departure times and flight lengths are parameters of considerable importance in the model, as both affect the turn time between each pair of flights. The single variable that is considered when calculating the departure time of each flight is the relative position of that flight within the flight block; the type of flight (local or cross-country) is irrelevant. This suggests that a specific departure time can be assigned to either a local or a cross-country flight. This dual assignment possibility creates a significant complexity in terms of the number of possible combinations of different schedules and turn times for a given number of flights. If there are $n$ local flights and $m$ cross-country flights, the number of possible schedules is

$$
N_{S}=\frac{(n+m) !}{n ! m !}
$$

Since cross-country flights are inherently longer than local flights, the departure time of a particular flight, along with that flight's length, creates possibilities of connections with other flights that must be examined. The sequence in which the flights are organized is therefore critical. It is therefore reasonable to embed the optimization model within a larger simulation model. The general idea is to, using a Monte-Carlo approach, run a number of optimization problems, each assuming a fixed number of flights with different sequences. The most desirable schedule then becomes that which minimizes overall turn times. Figure 2 shows the simplified flow diagram of the simulation model. 


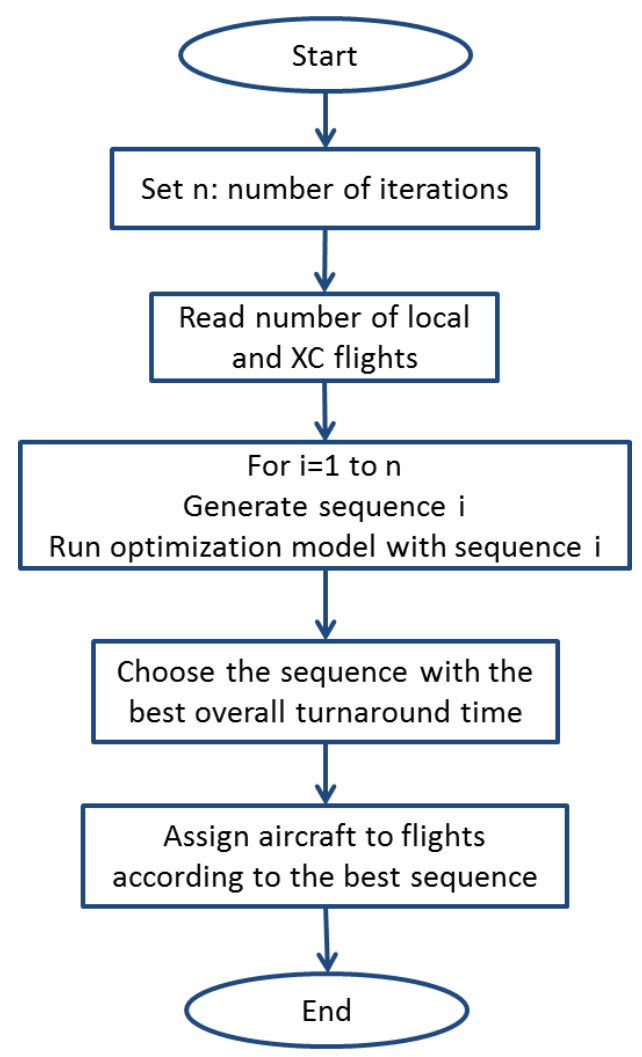

Figure 2. Simplified flow diagram of the simulation model.

Henceforth, the term "simulation model" will refer to the overall simulation model containing the embedded optimization algorithm.

\section{Assumptions of the Simulation Model}

With the intention of facilitating the formulation of a useful simulation model to accomplish the desired goal, the following assumptions were incorporated:

- Each flight block will accommodate a total of 13 flights.

- The flight dispatcher will know, prior to running the model, the number of crosscountry flights that will be operated in each block and the planned length of each flight.

- The number of local flights in each block will be calculated by subtracting the number of cross-country flights from the total number of flights.

- In each iteration of the simulation, a random sequence of all flights will be generated and optimized (in terms of the assignment of aircraft to specific flights).

\section{Flight Cancelling Process}

The number of available aircraft is one of the most important constraints in the problem because it limits the number of flights that can actually be operated. This constraint is dependent on maintenance operations. Currently, the flight dispatcher assigns flights to students according to the number of aircraft that the maintenance operation reports as available each day. Note that, while the maintenance scheduling process is conducted independently of flight scheduling and dispatching, maintenance scheduling needs to be considered when converting a theoretical schedule that assumes the availability of 16 aircraft into a feasible schedule that considers only those aircraft actually available. 
If the actual number of available aircraft is lower than the theoretical number of aircraft, an intuitive plan to convert the ideal schedule into a practical schedule would consider choosing, according to specific criteria, some of the aircraft and cancelling their assigned flights. At this point, the foremost question becomes how that choice is to be made. Because the goal of the research being conducted is to increase the utilization rate of the aircraft fleet, it is important that the selection of the aircraft that will be cancelled is in accordance with that goal.

A cancellation proposition algorithm was developed, an explanation of which follows in Table 1, through the use of a hypothetical example. The general principle underlying the algorithm is the sorting of theoretical aircraft according to a standardized weighted average of the number of local and cross-country flights, and the cancellation of aircraft with the least impact on the original schedule (in terms of the number of associated cancelled flights). The concept of weighting the number of local and cross-country flights is derived from the intent to minimize to the extent possible the number of cancelled local and cross-country flights. The weights used in the model indicate the respective priorities on local and cross-country flights, and may be varied accordingly.

\section{Test Plan and Implementation}

The implementation team for the project consisted of graduate and undergraduate student employees of the Advanced Aviation Analytics Institute for Research at the university. This team of researchers developed the scheduling, simulation, and cancellation algorithms into operable software, using the $\mathrm{R}$ language as a platform and a web-based interface to make the results available to dispatch personnel (Figure 3). The team proposed a pilot test focused on limited testing of the model and user interface to identify how to best incorporate the scheduling model into the dispatch operation with minimal disruption of existing processes. The pilot test phase provides an opportunity to test the usability of the interface by dispatchers, and to aid in the development of standard operating procedures for the use of the interface and of training procedures for dispatchers. Again, it is important to note that the reduction of turn times must be implemented in a manner that does not result in a negative impact on operational safety.

The schedule generated by the algorithm consists of a preliminary schedule in which local and cross-country flights are assigned to generic aircraft for each flight block throughout the day. For example, Aircraft 1 may be assigned to a local flight at 0730, a cross-country flight at 0930, and a local flight at 1330. Departure times are then assigned to these generic aircraft. The assignment of an actual aircraft to these generic aircraft results in the creation of the user schedule, and is made on the basis of a maintenance priority ranking that is determined daily by maintenance personnel. This maintenance priority exists to ensure that the aircraft with the earliest upcoming maintenance requirements are operated in such a manner that the associated hour limits are not exceeded, and is communicated to dispatch and operations personnel every morning before operations begin. 
Table 1. Computational Implementation of the Model

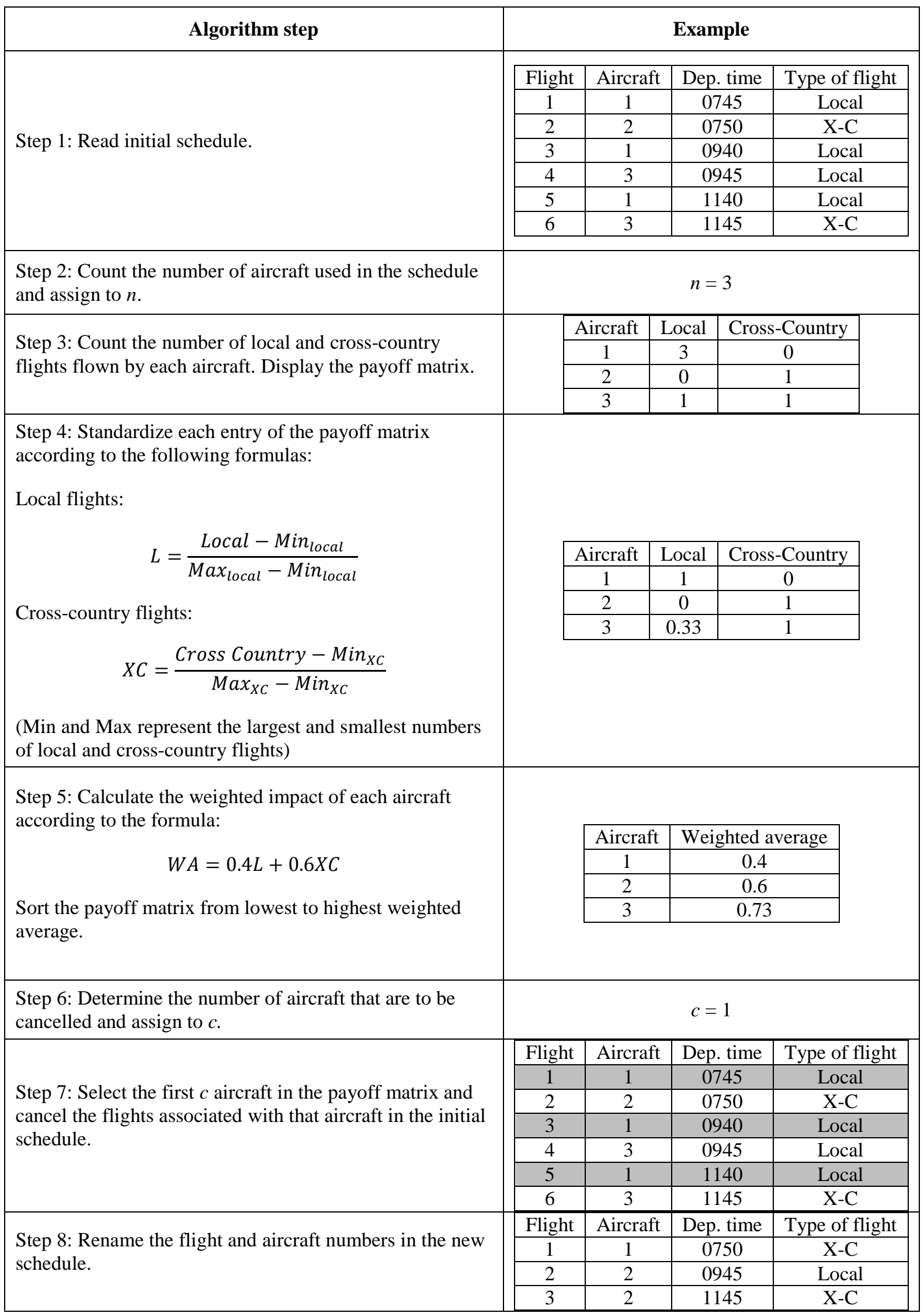




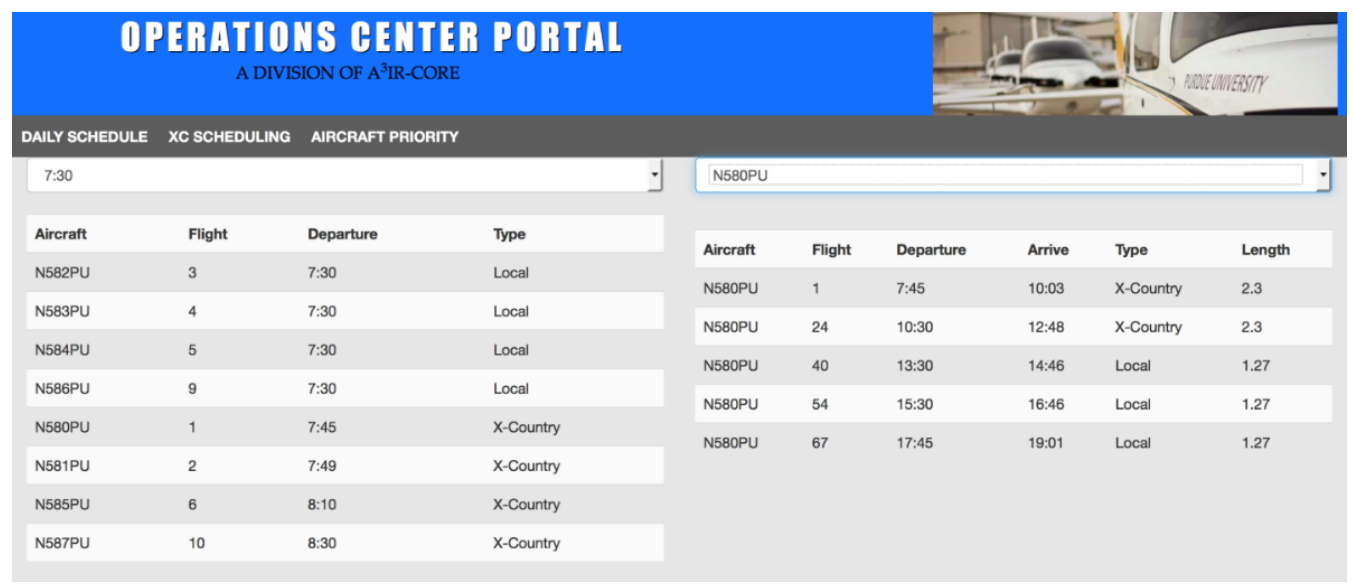

Figure 3. The web-based interface used to communicate the daily user schedule.

Challenges to implementation that were identified early in the process include those related to specified departure times, schedule management, and logistics. The identification of potential issues related to specification of departure times was conducted through the use of pre- and post-flight observations made by the research center team, and through discussions with flight instructors employed in the program. With regard to schedule management, challenges include establishing standards for management of the daily schedule during disruptions such as unscheduled weather situations or maintenance-related problems, providing an acceptable user interface that will allow dispatchers to make appropriate decisions based on the results of the model, and understanding effects on the dispatch process caused by the implementation itself.

The pilot test consisting of the implementation of only the sequencing portion of the scheduling model was conducted at the end of the Fall 2015 semester at the university. This test set the staggering of departures $\left(t_{s}\right)$ to 0 and involved ensuring only that scheduled flights remained in the proper sequence. The testing occurred over the first three flight blocks (0730, 0930, and 1130) over a three-day period at the end of the Fall 2015 semester, and involved the presence of a research center team member for the collection of observational data related to the implementation and to provide any necessary assistance to the dispatchers. The three objectives of the pilot test were as follows:

1. To observe and document any specific difficulties in using and managing the site from the perspective of the dispatcher in order to track errors that could potentially affect dispatch efficiency,

2. To obtain measurements of specific parameters applicable to the scheduling model in order to statistically evaluate the impact of the model on turn time, and 
3. To identify how best to incorporate the use of the scheduling interface through the dispatch role.

The components of the plan for the pilot test were the collection of information about scheduled cross-country flights, the actual running of the scheduling model, the assignment of aircraft to the generic aircraft output by the model, management of the schedule, and troubleshooting. Each of these elements is discussed in turn below.

Cross-country flights (which, by definition, cross the boundary of a single two-hour time block) are currently scheduled using a lottery system. This is handled by means of students who sign up for additional slots that are otherwise unoccupied on a so-called "daily sheet" (Figure 4).

\begin{tabular}{|c|c|c|c|c|}
\hline \multirow{7}{*}{$\begin{array}{l}\text { Ŝ } \\
\text { ㅇ } \\
\text { ஸे }\end{array}$} & \multicolumn{2}{|c|}{ Day of the Week: Friday } & $\begin{array}{c}\text { Date: } \mathbf{1 2 / 0 3 / 1 5} \\
1130\end{array}$ & $\begin{array}{r}\text { Week \#15 } \\
1330\end{array}$ \\
\hline & Student A & Student F & Student K & Student O \\
\hline & Student B & Student G & Student L & Student P \\
\hline & Student C & Student H & Student J & Student Q \\
\hline & Student D & Student I & Student M & Student R \\
\hline & Student E & Student A & Student N & Student S \\
\hline & & Student J & & Student $M$ \\
\hline
\end{tabular}

Figure 4. Daily sheet.

The slots shown in regular typeface are standard slots that are prescheduled for flight students. The italicized entries are previously unscheduled slots for which students desiring cross-country flights have registered and to which they have been assigned through the lottery process. In this example, Student A has a regular 0730 slot and an extra 0930 slot. The assumption is that the student will fly a cross-country flight, so that information would be used as an input to the schedule model. It should be noted that the schedule model implementation will obviate the need for the lottery process, since it will ensure that all requested cross-country flights are scheduled, which may result in the additional benefit of reducing the number of student completions that are potentially delayed due to the failure of students to obtain cross-country slots through the lottery system.

Aircraft are assigned to flight sequences after the model is run. Those aircraft that are out of service for maintenance during the assignment process are not included. As noted previously, aircraft are assigned to the generic sequence constructed by the scheduling algorithm after accounting for maintenance priorities, which are communicated to the dispatch operation through the use of a common display that contains maintenance information, 
including inspection status and time remaining until next inspection, for each available aircraft. Aircraft that have a limited number of hours remaining until their next maintenance event are not assigned to flight sequences that will exceed the number of flyable hours remaining in the current cycle.

Schedule management consists of ensuring that aircraft follow the scheduled sequence. Flight students will continue to be dispatched using existing operating procedures. The student is asked whether he or she is making a local or cross-country flight. If a local flight is to be made, the student is to be dispatched in the first available local aircraft listed on the user schedule (the model output adjusted for maintenance priority) within the two-hour block. If the student is to fly a cross-country flight, he or she is to be dispatched in the first available cross-country aircraft listed on the user schedule. Once the aircraft is assigned to the student, the aircraft is noted on the daily schedule as having been dispatched.

Troubleshooting and tactical changes may become necessary during the course of normal operations in certain circumstances. Such circumstances could include weather changes rendering conditions unflyable, aircraft that must be taken out of service for maintenance inspections or discrepancies during the day, aircraft that are returned to service from maintenance, or the reversion of a scheduled cross-country flight to a local flight. It is anticipated that changes of this nature will be handled by a small back office operations center staff tasked with strategic planning and tactical changes that may necessitate the rerunning of the scheduling model during a particular day's normal operating hours. This process would not modify students' schedules, but would simply reorder the assignment of aircraft to particular flights.

Logistical requirements of the pilot test included training the dispatchers who were to be involved in the test and briefing them appropriately on the objectives, standard procedures, and potential challenges that the test would involve. As noted, a member of the research center team was present during the testing period to answer questions, observe the process and its effects, and ensure that the applicable procedures were followed correctly.

\section{Results}

The results of the pilot test were examined through the use of a single metric: the cumulative times for each aircraft turn that occurred during the period. As was explained in a previous section, the objective function in the linear programming process is the overall turn time, so it is clear that the goal of the model is to minimize this quantity. The data for this metric were collected using an aircraft transponder-based data collection hardware and software platform that was designed for measurement and validation of fleet management metrics (McNamara, Mott, \& Bullock, 2016). 
For purposes of statistical analysis, the test period was compared with a three-day period that occurred at precisely the same point (a three-day period immediately before final exam week) during the spring semester of 2015. This minimizes variables that may impact the validity of the test to the extent possible. While the number of scheduled flights, weather conditions, and available aircraft may differ between the tests, the general frequency of flights during the two time periods is similar.

A conventional unpaired two-sample t-test between the turn time groups was run in Microsoft Excel. Unequal variances were assumed. The results are shown below in Table 2.

Table 2. t-Test: Two-Sample Assuming Unequal Variances

\begin{tabular}{lrr}
\hline & Variable 1 & Variable 2 \\
\hline Mean & 66.39117647 & 41.49313734 \\
Variance & 1158.804275 & 222.8768749 \\
Observations & 17 & 17 \\
Hypothesized Mean & \multicolumn{2}{|c}{} \\
Difference & 0 & \\
df & 22 & \\
t Stat & 2.761758438 \\
$\mathrm{P}(\mathrm{T}<=\mathrm{t})$ one-tail & 0.005690054 & \\
t Critical one-tail & 1.717144374 & \\
$\mathrm{P}(\mathrm{T}<=\mathrm{t})$ two-tail & 0.011380108 & \\
$\mathrm{t}$ Critical two-tail & 2.073873068 & \\
\hline
\end{tabular}

The means shown in Table 2 are in minutes. A two-tailed effect size of 0.947 was calculated using Cohen's $d$ test, resulting in a power value of 0.97 . It is evident that a decrease in mean turn times occurred post-implementation during the pilot test period. This decrease may be considered significant $(p=0.01)$. The results of the conventional two-tailed test were confirmed through a Bayesian one-way ANOVA formulated for nonhomogeneous residual variances and assuming an uninformed prior, which indicated a 95\% credible interval for the difference in means ranging from 5.12 to 42.6 minutes, with a mean difference of 23.7 minutes (Figure 5). The results are similarly evident from examination of a bar graph of the pre-implementation and post-implementation turn times (Figure 6). 


\section{Contrast between Pre- and Post-implementation Means}

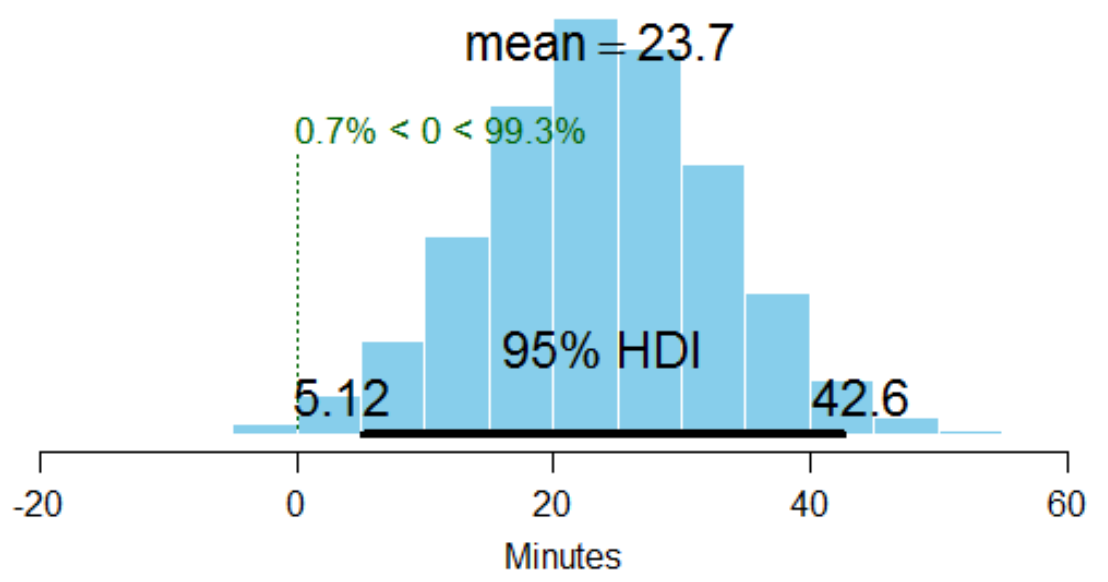

Figure 5. Credible difference between pre- and post-implementation means.

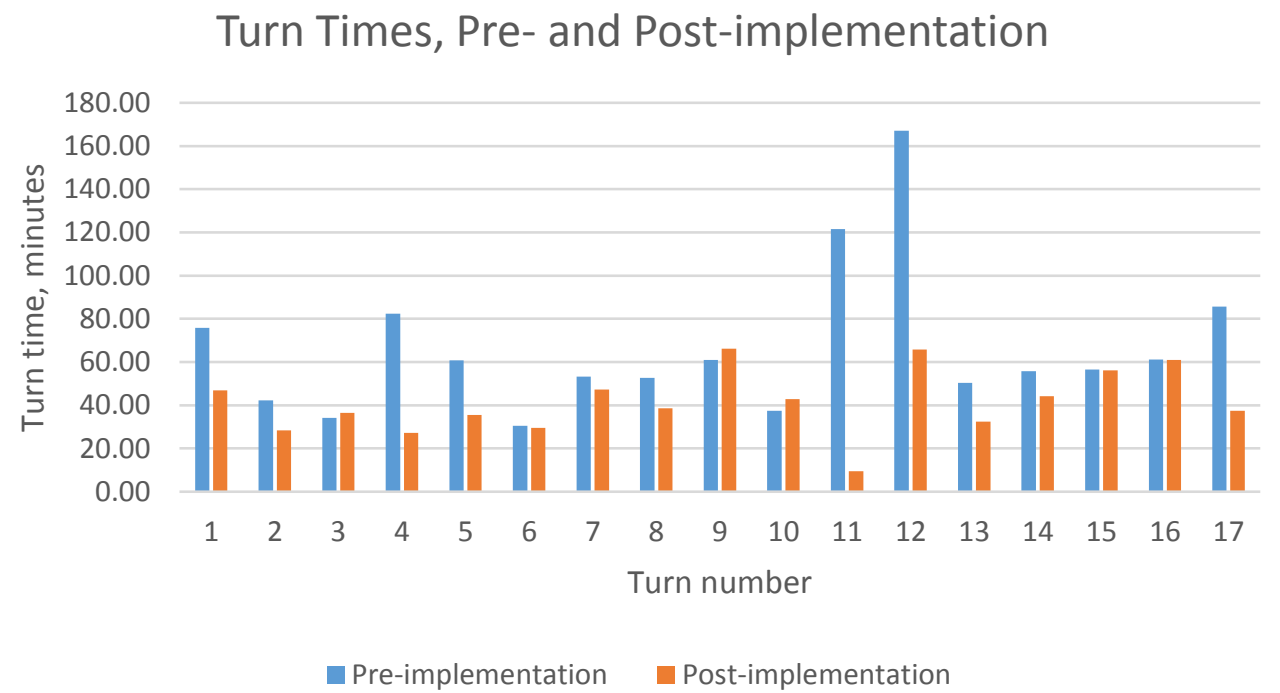

Figure 6. Graph of pre-implementation and post-implementation turn times.

\section{Conclusions}

Preliminary results from the pilot test indicate that the implementation of the new scheduling algorithm has resulted in improvements in the operational efficiency of the flight dispatch process. These results will be confirmed in further testing, which is ongoing. While the experimental design was intended to control for the effects of external variables to the greatest extent possible, it should be noted that the results may be influenced by factors other than the algorithm implementation. 
The experience of developing an optimization model for the student flight training scheduling process at the university during a period in which the number of enrolled students in the Professional Flight Program increased with respect to previous years while the number of aircraft remained constant indicates that optimization is a task that becomes especially relevant when resources are limited and an expectation of better use of those resources is prevalent.

The combination of simulation and optimization in this particular problem is an efficient way of taking advantage of the combinatorial complexity of the problem, and directing it to improve the optimization. The process of generating random sequences of flights, optimizing each one (in terms of the turn time) and then choosing the sequence with the best (minimum) turn time allows a more general perspective of the difficulty of generating an optimal flight schedule.

\section{Future Research}

With regard to non-program-specific research potential, there are certainly opportunities to apply the generalizable concepts suggested previously to other collegiate flight programs that seek improvements to utilization efficiency. It is hoped that such research will lead to improvements that will benefit all programs comprising the collegiate aviation community. 


\section{References}

Abara, J. (1989). Applying integer linear programming to the fleet assignment problem. Interfaces, 19(4), 20-28. doi:10.1287/inte.19.4.20

Avery, B. K. (2014). An analysis to determine the maximum utilization of Purdue University's aircraft fleet. (Unpublished capstone project proposal, Purdue University, West Lafayette, IN).

Bazargan, M. (2012). Airline operations and scheduling. Farnham, Surrey, UK: Ashgate Publishing, Ltd.

Bazargan, M., \& McGrath, R. N. (2003). Discrete event simulation to improve aircraft availability and maintainability. Paper presented at the IEEE 2003 Reliability and Maintainability Symposium, Tampa, FL.

Daniels, M. E. (2014). An open letter to the people of Purdue. Retrieved from http://www.purdue.edu/president/email/2014/1401-medopenletter.html

Daniels, M. E. (2015). An open letter to the people of Purdue. Retrieved from http://www.purdue.edu/president/messages/1501-med-openletter.html

Desaulniers, G., Desrosiers, J., Dumas, Y., Solomon, M. M., \& Soumis, F. (1997). Daily aircraft routing and scheduling. Management Science, 43(6), 841-855. doi:10.1287/mnsc.43.6.841

El Moudani, W., \& Mora-Camino, F. (2000). A dynamic approach for aircraft assignment and maintenance scheduling by airlines. Journal of Air Transport Management, 6(4), 233-237. doi:10.1016/s09696997(00)00011-9

Ferguson, A. R., \& Dantzig, G. B. (1954). The Problem of Routing Aircraft, a Mathematical Solution (RAND-P-561). Retrieved from http://www.dtic.mil/cgi-bin/GetTRDoc?AD=AD0604395

Ferguson, A. R., \& Dantzig, G. B. (1956). The allocation of aircraft to routesan example of linear programming under uncertain demand. Management Science, 3(1), 45-73. doi:10.1287/mnsc.3.1.45

Freling, R., Pinto Paixao, J. M., \& Wagelmans, A. P. M. (1995). Models and Algorithms for Vehicle Scheduling (EI 9562-/A). Retrieved from https://www.researchgate.net/profile/Albert_Wagelmans/publication/4 780260_Models_and_Algorithms_for_Vehicle_Scheduling/links/0f317 53721743ebfae000000.pdf 
Grönkvist, M. (2005). The tail assignment problem. (Doctoral dissertation, Chalmers University of Technology and Goteborg University, Goteborg, Sweden). Retrieved from http://citeseerx.ist.psu.edu/viewdoc/download?doi=10.1.1.103.825\&re $\mathrm{p}=\mathrm{rep} 1 \&$ type $=$ pdf

Hane, C. A., Barnhart, C., Johnson, E. L., Marsten, R. E., Nemhauser, G. L., $\&$ Sigismondi, G. (1995). The fleet assignment problem: Solving a large-scale integer program. Mathematical Programming, 70(1-3), 211-232. doi:10.1007/bf01585938

Jacobs, R. S. (2014). Optimization of daily flight training schedules. (Master's thesis, Naval Postgraduate School, Monterey, CA). Retrieved from http://www.dtic.mil/cgi-bin/GetTRDoc?AD=ADA607792

Jarrah, A. I., Goodstein, J., \& Narasimhan, R. (2000). An efficient airline refleeting model for the incremental modification of planned fleet assignments. Transportation Science, 34(4), 349-363. doi:10.1287/trsc.34.4.349.12324

McNamara, M., Mott, J., \& Bullock, D. (2016). Leveraging aircraft avionics for fleet and airport management. Transportation Research Record: Journal of the Transportation Research Board (2569), 32-41. doi:10.3141/2569-04

Mott, J. H., \& Bullock, D. M. (2015). Improving training aircraft utilization in collegiate flight programs: A case study at Purdue University. Collegiate Aviation Review, 33(1).

Salazar-González, J.-J. (2014). Approaches to solve the fleet-assignment, aircraft-routing, crew-pairing and crew-rostering problems of a regional carrier. Omega, 43, 71-82.

Subramanian, R., Scheff, R. P., Quillinan, J. D., Wiper, D. S., \& Marsten, R. E. (1994). Coldstart: fleet assignment at Delta Air Lines. Interfaces, 24(1), 104-120. doi:10.1287/inte.24.1.104

Yao, Y., Zhao, W., Ergun, O., \& Johnson, E. (2005). Crew pairing and aircraft routing for on-demand aviation with time window. Available at SSRN 822265. doi:10.2139/ssrn.822265 
Mott et al.: Increasing Collegiate Flight Training Fleet Utilization

Zhu, X. (2006). Discrete two-stage stochastic mixed-integer programs with applications to airline fleet assignment and workforce planning problems. (Doctoral dissertation, Virginia Polytechnic Institute and State University, Blacksburg, VA). Retrieved from https://vtechworks.lib.vt.edu/bitstream/handle/10919/27212/dissertatio n.pdf?sequence $=1 \&$ isAllowed $=y$ 